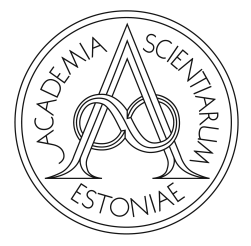

Proceedings of the Estonian Academy of Sciences,

2022, 71, 1, 103-116

https://doi.org/10.3176/proc.2022.1.09

NANOMECHANICS

Available online at www.eap.ee/proceedings

\title{
Free vibrations of stepped nano-beams with cracks
}

\author{
Jaan Lellep* and Artur Lenbaum \\ Institute of Mathematics and Statistics, University of Tartu, Narva mnt 18, 51009 Tartu, Estonia \\ Received 11 November 2021, accepted 14 January 2022, available online 16 February 2022
}

(C) 2022 Authors. This is an Open Access article distributed under the terms and conditions of the Creative Commons Attribution 4.0 International License CC BY 4.0 (http://creativecommons.org/licenses/by/4.0).

\begin{abstract}
Free vibrations of stepped beams made of nano-materials are investigated. The nano-beams under consideration have piecewise constant dimensions of the cross section and are weakened with crack-like defects. The influence of the crack on the mechanical behaviour of the nano-beam is defined with the aid of the additional local compliance. Mechanical vibrations of the nano-beam are specified in the non-local theory of elasticity developed by Eringen. Numerical results are presented for nano-beams with two different thicknesses.
\end{abstract}

Key words: nano-beam, crack, eigenfrequency.

\section{INTRODUCTION}

In the classical theory of elasticity the Hooke's law states that stress and strain tensors are proportional at any point of the body. However, it is accepted that for nano-materials one can use non-local constitutive laws (see Eringen [11], Reddy [19], Lu et al. [17]). Although the principles of non-local elasticity were developed more than thirty years ago, the intensive investigation of the behaviour of nano-beams and nanoplates started quite recently (see Reddy [19], Pradhan and Phadikar [18], Challamel [7]). Challamel [6] employed strain gradient elasticity and non-local elasticity models combined with the variational approach in order to derive non-local higher-order shear theories. Adali [1] and Wang et al. [23] presented the buckling analysis of nano-rods based on the non-local Timoshenko theory of beams. Challamel and Elishakoff [8] demonstrated on the basis of the Timoshenko theory that the surface stress effects may induce softening in the case of buckling problems.

In the present paper the non-local constitutive law is employed for the investigation of stepped nanobeams with defects. The aim of the paper is to elucidate the influence of cracks or crack-like defects on the natural frequencies of nano-beams. The influence of the crack on the vibration of the nano-beams is modelled with the aid of a massless rotating spring connecting the adjacent segments of the nano-beam.

\footnotetext{
*Corresponding author, jaan.lellep@ut.ee
} 


\section{FORMULATION OF THE PROBLEM}

In this section the free vibration of nano-beams will be studied. The nano-beam has stepped cross sections. For the sake of conciseness it is assumed that the cross section of the beam is of rectangular shape with width $b$ and height $h_{j}$ for $x \in\left(a_{j}, a_{j+1}\right)$. Here $j=0,1, \ldots, n$ and $a_{j}=0, a_{j+1}=l$. The origin of the coordinate axis $O x$ is located at the centre of the left-hand end of the nano-beam.

The nano-beam under consideration is weakened with stable crack-like defects. Let the extension of the crack located at $x=a_{j}$ be $c_{j}(j=1, \ldots, n)$ and the crack surface area

$$
S_{c j}=c_{j} b
$$

It is widely recognized that the defects deteriorate the mechanical behaviour of structural elements. Thus, it is important to know how the defects affect the natural frequencies of nano-beams.

This study establishes the eigenfrequencies for free vibrations of stepped nano-beams with cracks and analyses the sensitivity of eigenfrequencies on the geometrical and physical parameters of the nano-beam. Among these parameters the coordinates of the injured cross sections and dimensions of these sections are of primary importance.

\section{CONSTITUTIVE EQUATIONS}

It is expected that the mechanical behaviour of the nano-material is specified in the non-local theory of elasticity developed by Eringen [11]. In the non-local theory of elasticity the stress state $\sigma_{i j}^{n}(x)$ at a fixed point of the body $x \in V$ depends on the deformation state at each point $y$ of the body. According to Eringen [11], the non-local stress components can be defined as (here $i, j=1,2,3$ )

$$
\sigma_{i j}^{n}(x)=\iiint_{(V)} \mathrm{K}(|x-y|, \tau) \sigma_{i j}^{c}(y) d V .
$$

Here $\mathrm{K}$ denotes a kernel function which may have different forms whereas $\tau$ is a physical constant and $\sigma_{i j}^{c}$ refers to the classical stress tensor. The relationship between classical stresses and strain components is furnished by the well-known Hooke's law.

Evidently, different forms of the kernel $\mathrm{K}$ lead to different models of non-local elasticity. Eringen [11], Lu et al. [17] suggested that the kernel K be defined as the Green's function of a linear differential operator, or

$$
\mathscr{L} \mathrm{K}|x-y|=\delta(|x-y|),
$$

where $\delta$ represents the Dirac's function. In the two-dimensional case

$$
\mathscr{L} \mathrm{K}=\mathrm{K}-\eta \nabla^{2} \mathrm{~K}
$$

where $\nabla$ is the Laplacian operator. In (4) $\eta=\left(e_{0} a\right)^{2}, e_{0}$ and $a$ are the material constants (see Lu et al. [17]). The constant $a$ can be treated as the dimension of the cristalline lattice of the solid material. However, $e_{0}$ is a constant for adjusting the non-local theory to match the results of experiments. It has been verified by Eringen [11] that the constitutive model (4) provides an excellent coincidence of the dispersion curves of plane waves with the results of atomic lattice dynamics at lower wave numbers. Due to the relative simplicity of the model (4), it has been used in many studies on the vibration of nanostructural elements (see Ghannadpour et al. [12], Lu et al. [17], Roostai and Haghpanahi [20]).

It is well known that in the classical theory of elasticity the governing equations can be presented as

$$
\sigma_{i j}^{c}=\lambda \varepsilon_{k k} \delta_{i j}+\mu \varepsilon_{i j},
$$


where $\varepsilon_{i j}$ represents the deformation tensor, $\lambda$ and $\mu$ are the constants of Lame. These are coupled with the Young modulus $E$ and the Poisson ratio $v$ as

$$
E=\frac{\mu(3 \lambda+2 \mu)}{\lambda+\mu}, v=\frac{\lambda}{2(\lambda+\mu)}
$$

Eringen [11] suggested that the constitutive equations be used in the non-local thoery of elasticity as

$$
\sigma_{i j}^{n}-\eta \nabla^{2} \sigma_{i j}^{n}=\lambda \varepsilon_{k k} \delta_{i j}+\mu \varepsilon_{i j}
$$

\section{GOVERNING EQUATIONS IN GENERALIZED STRESSES}

The classical beam theory uses instead of the normal stress $\sigma$ the generalized stresses called the bending moment

$$
M=\iint_{(S)} \sigma z d S
$$

and the membrane force

$$
N=\iint_{(S)} \sigma d S
$$

Here $S$ refers to the cross-sectional area of the beam. It is reasonable to employ these variables in the nonlocal theory of elasticity, as well. In the classical beam theory the bending moment reads as (see Reddy [19])

$$
M_{c}=-E I \frac{\partial^{2} w}{\partial x^{2}} .
$$

In (10) $w$ denotes the transverse deflection (the vertical displacement of a point lying at the neutral axis of the beam) and $I$ is the moment of inertia of the cross section defined as

$$
I=\iint_{(S)} z^{2} d S .
$$

Evidently, in the case of a rectangular cross section with width $b$ and height $h$, the moment of inertia (the second moment) calculated from (11) becomes

$$
I=\frac{b h^{3}}{12} .
$$

Combining (5) and (7) results in

$$
\sigma_{i j}^{n}-\eta \nabla^{2} \sigma_{i j}^{n}=\sigma_{i j}^{c} .
$$

Let us treat the case $i=j=1$ (13) in detail. Multiplying (13) by width $z$ and integrating it over the cross section of the beam and considering (8)-(11), one obtains

$$
M-\eta \frac{\partial^{2} M}{\partial x^{2}}=-E I \frac{\partial^{2} w}{\partial x^{2}} .
$$

Integrating (13) over the cross section $S$ and making use of (9) leads to 


$$
N-\eta \frac{\partial^{2} N}{\partial x^{2}}=-E I^{1} \frac{\partial^{2} w}{\partial x^{2}}
$$

where $I^{1}$ refers to the first moment of the cross section defined as

$$
I^{1}=\iint_{(S)} z d S .
$$

Let prims denote the differentiation with respect to the coordinate $x$ and dots with respect to time $t$. Thus,

$$
\begin{gathered}
w^{\prime \prime}=\frac{\partial^{2} w}{\partial x^{2}}, \\
\ddot{w}=\frac{\partial^{2} w}{\partial t^{2}} .
\end{gathered}
$$

Making use of the notation (17), the equilibrium equations of a beam element can be presented as (see Soedel [21])

$$
N^{\prime}=\mu \ddot{u}
$$

and

$$
\begin{gathered}
M^{\prime}=Q, \\
Q^{\prime}=-N w^{\prime \prime}+\mu \ddot{w} .
\end{gathered}
$$

In (18), (19) $\mu$ denotes the mass per unit length of the beam, $Q$ is the shear force and $u$ represents the axial displacement.

It is assumed that the quantities $\mu$ and $I$ are piecewise constants. Thus, $I=I_{j}$ and $\mu=\mu_{j}$ for $x \in$ $\left(a_{j}, a_{j+1}\right)$, where $j=0, \ldots, n$. Eliminating the shear force $Q$ from the system (19) leads to the equation

$$
M^{\prime \prime}=-N w^{\prime \prime}+\mu_{j} \ddot{w}
$$

for $x \in\left(a_{j}, a_{j+1}\right) ; j=0, \ldots, l$.

The system (14), (20) can be transformed into the form of the fourth order equation

$$
\left(\eta N+E I_{j}\right) w^{I V}+\mu_{j}\left(\ddot{w}-\eta \ddot{w}^{\prime \prime}\right)-N w^{\prime \prime}=0
$$

for $x \in\left(a_{j}, a_{j+1}\right) ; j=0, \ldots, n$.

The bending moment (14) can be expressed via the vertical displacement $w$ as

$$
M=-\left(\eta N+E I_{j}\right) w^{\prime \prime}+\eta \mu_{j} \ddot{w}
$$

for each $x \in\left(a_{j}, a_{j+1}\right) ; j=0, \ldots, n$.

\section{MODELLING THE CRACK}

The fact that cracks and other defects affect the eigenfrequencies of structural elements was recognized long ago. In the present paper as in previous studies of the authors [16], the cracks are treated as additional sources of structural compliance. Following Dimarogonas [10], also Chondros et al. [9], it is assumed that the slope of the deflection has finite jumps at the cross sections with cracks and that the finite jump $\Delta_{j}$ reads as

$$
\Delta_{j}=C_{0 j} M\left(a_{j}, t\right) .
$$


Here $C_{0 j}$ denotes the additional compliance due to the crack at $x=a_{j} ; j=1, \ldots, n$ and

$$
\Delta_{j}=w^{\prime}\left(a_{j}+0, t\right)-w^{\prime}\left(a_{j}-0, t\right) .
$$

Let the stress intensity factor of a crack of length $c_{j}$ located at $x=a_{j}$ be $K_{j}$. It is recognized in the linear elastic fracture mechanics that

$$
G_{j}=\frac{K_{j}^{2}}{E^{\prime}}
$$

where $G_{j}$ represents the energy release rate, and $E^{\prime}=E$ in the case of plane stress state and $E^{\prime}=E /\left(1-v^{2}\right)$ in the case of plane strain state (see Anderson [2] and Broek [4]). On the other hand, it is known in linear fracture mechanics that the energy release rate $G$, the crack surface area $S$ and the compliance $C$ are coupled with the generalized force $P$ as (see Broek [4])

$$
G=\frac{P^{2}}{2} \frac{d C}{d S} .
$$

In the present case the generalized force is the bending moment applied at the cross section with a crack. This means that one can take

$$
G_{j}=\frac{M_{j}^{2}}{2 b} \frac{d C_{0 j}}{d c_{j}}
$$

where (1) is taken into account and $M_{j}=M\left(a_{j}, t\right) ; j=1, \ldots, n$. It was shown in the previous studies (see Lellep and Lenbaum [16], Lellep and Kraav [14], Lellep and Kägo [15]) that after specifying the stress intensity factor $K_{j}$ in (25), the relations (25), (27) result in

$$
C_{0 j}=\frac{72 \pi\left(1-v^{2}\right)}{E b h_{0 j}^{2}} f\left(s_{j}\right)
$$

where

$$
f\left(s_{j}\right)=\int_{0}^{s_{j}} \xi F^{2}(\xi) d \xi
$$

Here $s_{j}=c_{j} / h_{0 j}$ and $h_{0 j}=\min \left(h_{j-1}, h_{j}\right)$ for $j=1, \ldots, n$. The function $F=F(\xi)$ in (29) presents for $\xi=s_{j}$ the shape factor or correction factor. The correction factor is to be determined experimentally. However, in the present paper we are resorting to the existing data in literature (see Tada et al.[22]). According to [22], the correction factor can be taken as

$$
F(s)=1.93-3.07 s+14.53 s^{2}-25.11 s^{3}+25.80 s^{4}
$$

or for larger cracks

$$
F(s)=\left\{\begin{array}{l}
1.99-2.47 s+12.97 s^{2}-23.17 s^{3}+24.8 s^{4}, \quad s<0.5, \\
0.663(1-s)^{-3 / 2}, \quad 0.5 \leqslant s<1 .
\end{array}\right.
$$

Various interpolations of test results leading to different forms of the stress correction factors can be found in literature (see Tada et al. [22], Anderson [2]). A comparison of different approximations is presented in the paper by Caddemi and Calio [5].

Generally speaking, an element with a cracked section can be loaded by membrane forces or bending moments or by the combination of tension and bending. In the general case the stress intensity factor can be integrated as (see Hellan [13], Anderson [2]) 


$$
K=K_{M} F_{M}(s)+K_{N} F_{N}(s) .
$$

The approximation (30), (31) corresponds to the case where the stretching can be neglected and $F_{N}=0$ in (32). However, with regard to the problems of buckling of cracked columns, the last term in (32) is essential (see Zhou and Huang [24], Anifantis and Dimarogonas [3]). Thus, in the present case the jump of the slope of the deflection is given by (23), where the additional compliance $C_{0 j}$ is defined by (28)-(31). Substituting $M\left(a_{j}, t\right)$ into (23) according to (22), one obtains

$$
\Delta_{j}=C_{0 j}\left(-\left(\eta N+E I_{j}\right) w^{\prime \prime}\left(a_{j}+0, t\right)+\eta \mu_{j} \ddot{w}\left(a_{j}+0, t\right)\right)
$$

for $j=1, \ldots, n$. The jump conditions (33) with (24) can be presented as

$$
\begin{gathered}
w^{\prime}\left(a_{j}+0, t\right)=w^{\prime}\left(a_{j}-0, t\right) \\
+C_{0 j}\left(-\left(\eta N+E I_{j}\right) w^{\prime \prime}\left(a_{j}+0, t\right)+\eta \mu_{j} \ddot{w}\left(a_{j}+0, t\right)\right) .
\end{gathered}
$$

\section{SOLUTION TO THE EQUATION OF MOTION}

In order to solve the fourth order equation with partial derivatives (21), it is assumed that for each $t$ and $x \in\left(a_{j}, a_{j+i}\right)$ the displacement $w$ can be presented as

$$
w(x, t)=X_{j}(x) T(t)
$$

where $j=0, \ldots, n$. In the right-hand side of the equation (35) the functions $X_{j}(x)$ and $T(t)$ are the functions of a single variable only. It easily follows from (35) that

$$
\begin{aligned}
& \frac{\partial^{2} w}{\partial x^{2}}=X_{j}^{\prime \prime}(x) T(t), \\
& \frac{\partial^{4} w}{\partial x^{4}}=X_{j}^{\prime \prime \prime \prime}(x) T(t), \\
& \frac{\partial^{2} w}{\partial t^{2}}=X_{j}^{\prime \prime}(x) \ddot{T}(t), \\
& \frac{\partial^{4} w}{\partial x^{2} \partial t^{2}}=X_{j}^{\prime \prime}(x) \ddot{T}(t)
\end{aligned}
$$

for $x \in\left(a_{j}, a_{j+1}\right) ; j=0, \ldots, n$.

Substituting (36) into (21), one obtains the equation

$$
\left(\eta N+E I_{j}\right) X_{j}^{\prime \prime \prime \prime} T+\mu_{j}\left(X_{j} \ddot{T}-\eta X_{j}^{\prime \prime} \ddot{T}\right)-N X_{j}^{\prime \prime} T=0
$$

for $x \in\left(a_{j}, a_{j+1}\right) ; j=0, \ldots, n$.

The separation of variables in (37) leads to relations

$$
\frac{\ddot{T}}{T}=-\omega^{2}
$$

and

$$
\frac{\left(\eta N+E I_{j}\right) X_{j}^{\prime \prime \prime \prime}-N X_{j}^{\prime \prime}}{\mu_{j}\left(\eta X_{j}^{\prime \prime}-X_{j}\right)}=-\omega^{2},
$$

where $\omega$ denotes the frequency of natural vibrations. The last equality leads to the fourth order linear equation 


$$
\left(\eta N+E I_{j}\right) X_{j}^{\prime \prime \prime \prime}-\left(N-\eta \mu_{j} \omega^{2}\right) X_{j}^{\prime \prime}-\mu_{j} \omega^{2} X_{j}=0,
$$

which is to be solved for $x \in\left(a_{j}, a_{j+1}\right) ; j=0, \ldots, n$. The characteristic equation corresponding to (40) is

$$
\left(\eta N+E I_{j}\right) \lambda_{j}^{4}+\lambda_{j}^{2}\left(\eta \mu_{j} \omega^{2}-N\right)-\mu_{j} \omega^{2}=0 .
$$

The biquadratic equation (41) can be easily solved; the roots are

$$
\lambda_{j 1}=v_{j} ; \quad \lambda_{j 2}=-v_{j} ; \quad \lambda_{j 3}=i \beta_{j} ; \quad \lambda_{j 4}=-i \beta_{j},
$$

where $i$ is the imaginary unit and

$$
v_{j}=\sqrt{\frac{N-\mu_{j} \eta \omega^{2}+R_{j}}{2\left(\eta N+E I_{j}\right)}}
$$

while

$$
\beta_{j}=\sqrt{\frac{-N+\mu_{j} \eta \omega^{2}+R_{j}}{2\left(\eta N+E I_{j}\right)}} .
$$

In (43), (44) the following notation is used:

$$
R_{j}=\sqrt{\left(N-\mu_{j} \eta \omega^{2}\right)^{2}+4 \mu_{j} \omega^{2}\left(\eta N+E I_{j}\right)} .
$$

The roots of the characteristic equation presented by (42)-(45) allow to present the general solution to the fourth order equation (40) as

$$
X_{j}(x)=A_{j} \cosh v_{j} x+B_{j} \sinh v_{j} x+C_{j} \cos \beta_{j} x+D_{j} \sin \beta_{j} x,
$$

where $j=0, \ldots, n$. The general solution to the second order equation (38) is

$$
T(t)=\bar{A}_{1} \cos \omega t+\bar{A}_{2} \sin \omega t .
$$

It is reasonable to assume that at the initial moment $t=0$ the nano-beam is not yet deformed. Thus, $T(0)=0$ and according to (47), $\bar{A}_{1}=0$. This means that one can present the deflection as

$$
w(x, t)=X(x) \sin \omega t
$$

for $x \in\left(a_{j}, a_{j+1}\right) ; \quad j=0, \ldots, n$. It is worthwhile to mention that the coefficient $\bar{A}_{2}=0$ is neglected in (48) as $X_{j}$ involves, according to (46), four arbitrary constants and a new coefficient cannot introduce more generality. As regards the constants $A_{j}, B_{j}, C_{j}, D_{j}$, they will be defined making use of the corresponding boundary conditions

$$
X_{0}(0)=0 ; X_{0}^{\prime}(0)=0
$$

and

$$
\begin{aligned}
& -\left(\eta N+E I_{n}\right) X_{n}^{\prime \prime}(l)-\mu \eta \lambda^{2} X_{n}(l)=0 \\
& -\left(\eta N+E I_{n}\right) X_{n}^{\prime \prime \prime}(l)-\mu \eta \lambda^{2} X_{n}^{\prime}(l)=0
\end{aligned}
$$

as well as the jump conditions (34). 


\section{THE CONTINUITY AND JUMP CONDITIONS}

Due to the physical considerations it is evident that displacements of the central line of the nano-beam are continuous everywhere within the segment $(0, l)$. Otherwise the nano-beam should be broken. The continuity of deflection $w$ at $x=a_{j}$ means according to (48) that

$$
X_{j}\left(a_{j}\right)=X_{j-1}\left(a_{j}\right) .
$$

However, the slope of the deflection is not continuous at $x=a_{j}$. The finite jumps of the slope are defined by (34). Making use of (46), (48), one can present (34) as

$$
X_{j-1}^{\prime}\left(a_{j}\right)=X_{j}^{\prime}\left(a_{j}\right)+C_{0 j} \eta \mu_{j} \omega^{2} X_{j}\left(a_{j}\right)+C_{0 j}\left(\eta N+E I_{j}\right) X_{j}^{\prime \prime}\left(a_{j}\right) .
$$

Evidently, the bending moment $M$ is continuous everywhere including $x=a_{j}$. Thus, according to (22), (48)

$$
\begin{gathered}
\left(\eta N+E I_{j}\right) X_{j}^{\prime \prime}\left(a_{j}\right)+\eta \mu_{j} \omega^{2} X_{j}\left(a_{j}\right) \\
=\left(\eta N+E I_{j-1}\right) X_{j-1}^{\prime \prime}\left(a_{j}\right)+\eta \mu_{j-1} \omega^{2} X_{j-1}\left(a_{j}\right),
\end{gathered}
$$

where $j=1, \ldots, n$.

Due to the continuity of the shear force $Q=M^{\prime}$ where $M$ is defined by (22), one has

$$
\begin{gathered}
\left(\eta N+E I_{j}\right) X_{j}^{\prime \prime \prime}\left(a_{j}\right)+\eta \mu_{j} \omega^{2} X_{j}^{\prime}\left(a_{j}\right) \\
=\left(\eta N+E I_{j-1}\right) X_{j-1}^{\prime \prime \prime}\left(a_{j}\right)+\eta \mu_{j-1} \omega^{2} X_{j-1}^{\prime}\left(a_{j}\right)
\end{gathered}
$$

for $j=1, \ldots, n$.

\section{NATURAL FREQUENCIES OF THE CANTILEVER NANO-BEAM}

In order to determine the natural frequencies of the nano-beam, one has to use the mode forms (46) for $x \in\left(a_{j}, a_{j+1}\right) ; j=0, \ldots, n$. The function $X_{j}(x) ; j=0, \ldots, n$ must satisfy the boundary requirements (49), (50) as well as continuity and jump conditions (51)-(54). The conditions (49)-(54) can be interpreted as linear homogeneous algebraic equations with respect to unknowns $A_{j}, B_{j}, C_{j}, D_{j} ; j=0, \ldots, n$. Evidently, this system has non-trivial solutions if and only if its determinant $\Delta$ equals zero. Let us consider the particular case if $n=1$ in greater detail. In this case the nano-beam has a single step at $x=a$ (the subscript of $a_{1}$ can be omitted herein).

It can be easily rechecked that the boundary conditions (49) result in

$$
\begin{gathered}
A_{0}=-C_{0}, \\
B_{0}=-\frac{\beta_{0}}{v_{0}} D_{0} .
\end{gathered}
$$

Thus, it follows from (46) and (55) that

$$
X_{0}=A_{0}\left(\cosh v_{0} x-\cos \beta_{0} x\right)+B_{0}\left(\sinh v_{0} x-\frac{v_{0}}{\beta_{0}} \sin \beta_{0} x\right),
$$

which is valid for $x \in(0, a)$.

The continuity requirement (51) takes for $j=1$ the form

$$
\begin{gathered}
A_{1} \cosh v_{1} a+B_{1} \sinh v_{1} a+C_{1} \cos \beta_{1} a+D_{1} \sin \beta_{1} a \\
-A_{0}\left(\cosh v_{0} a-\cos \beta_{0} a\right)-B_{0}\left(\sinh v_{0} a-\frac{v_{0}}{\beta_{0}} \sin \beta_{0} a\right)=0 .
\end{gathered}
$$

Making use of (46), (56), one can put the jump conditions (52) into the form (here $C_{01}$ is replaced by $C$ ) 


$$
\begin{aligned}
& A_{1}\left\{v_{1} \sinh v_{1} a+C\left(\eta \mu_{1} \omega^{2}+v_{1}^{2}\left(\eta N+E I_{1}\right) \cosh v_{1} a\right)\right\} \\
& +B_{1}\left\{v_{1} \cosh v_{1} a+C\left(\eta \mu_{1} \omega^{2}+v_{1}^{2}\left(\eta N+E I_{1}\right) \sinh v_{1} a\right)\right\} \\
& +C_{1}\left\{-\beta \sin \beta_{1} a+C\left(\eta \mu_{1} \omega^{2}-\beta_{1}^{2}\left(\eta N+E I_{1}\right) \cos \beta_{1} a\right)\right\} \\
& +D_{1}\left\{\beta \cos \beta_{1} a+C\left(\eta \mu_{1} \omega^{2}-\beta_{1}^{2}\left(\eta N+E I_{1}\right) \sin \beta_{1} a\right)\right\} \\
& -A_{0}\left(v_{0} \sinh v_{0} a+\beta_{0} \sin \beta_{0} a\right)-v_{0} B_{0}\left(\cosh v_{0} a-\cos \beta_{0} a\right)=0 .
\end{aligned}
$$

The continuity condition (53) takes the form

$$
\begin{gathered}
A_{1}\left(\left(\eta N+E I_{1}\right) v_{1}^{2}+\eta \mu_{1} \omega^{2}\right) \cosh v_{1} a \\
+B_{1}\left(\left(\eta N+E I_{1}\right) v_{1}^{2}+\eta \mu_{1} \omega^{2}\right) \sinh v_{1} a \\
+C_{1}\left(-\left(\eta N+E I_{1}\right) \beta_{1}^{2}+\eta \mu_{1} \omega^{2}\right) \cos \beta_{1} a \\
+D_{1}\left(-\left(\eta N+E I_{1}\right) \beta_{1}^{2}+\eta \mu_{1} \omega^{2}\right) \sin \beta_{1} a \\
-A_{0}\left\{\left(\eta N+E I_{0}\right)\left(v_{0}^{2} \cosh v_{0} a+\beta_{0}^{2} \cos \beta_{0} a\right)+\eta \mu_{0} \omega^{2}\left(\cosh v_{0} a-\cos \beta_{0} a\right)\right\} \\
-B_{0}\left(v_{0}\left(\eta N+E I_{0}\right)\left(v_{0} \sinh v_{0} a+\beta_{0} \sin \beta_{0} a\right)+\eta \mu_{0} \omega^{2}\left(\sinh v_{0} a\right.\right. \\
\left.\left.-\frac{v_{0}}{\beta_{0}} \sin \beta_{0} a\right)\right)=0 .
\end{gathered}
$$

Finally, the requirement (54) transforms into

$$
\begin{gathered}
A_{1}\left(v_{1}^{3}\left(\eta N+E I_{1}\right)+\eta \mu_{1} \omega^{2} v_{1}\right) \sinh v_{1} a \\
+B_{1}\left(v_{1}^{3}\left(\eta N+E I_{1}\right)+\eta \mu_{1} \omega^{2} v_{1}\right) \cosh v_{1} a \\
+C_{1}\left(\beta_{1}^{3}\left(\eta N+E I_{1}\right)-\eta \mu_{1} \omega^{2} \beta_{1}\right) \sin \beta_{1} a \\
+D_{1}\left(-\beta_{1}^{3}\left(\eta N+E I_{1}\right)+\eta \mu_{1} \omega^{2} \beta_{1}\right) \cos \beta_{1} a \\
-A_{0}\left(\left(\eta N+E I_{0}\right)\left(v_{0}^{3} \sinh v_{0} a+\beta_{0}^{3} \sin \beta_{0} a\right)\right. \\
\left.+\eta \mu_{0} \omega^{2}\left(v_{0} \sinh v_{0} a-\beta_{0} \sin \beta_{0} a\right)\right) \\
-B_{0}\left(\left(\eta N+E I_{0}\right)\left(v_{0}^{3} \cosh v_{0} a+\beta_{0}^{2} v_{0} \cos \beta_{0} a\right)\right. \\
\left.+\eta \mu_{0} \omega^{2} v_{0}\left(\cosh v_{0} a-\cos \beta_{0} a\right)\right)=0 .
\end{gathered}
$$

The boundary conditions (50) can be presented with the help of (46) as (here $n=1$ )

$$
\begin{gathered}
-\left(\eta N+E I_{1}\right) v_{1}^{2}\left(A_{1} \cosh v_{1} l+B_{1} \sinh v_{1} l\right)-\beta_{1}^{2}\left(C_{1} \cos \beta_{1} l+D_{1} \sin \beta_{1} l\right) \\
-\mu \eta \omega^{2}\left(A_{1} \cosh v_{1} l+B_{1} \sinh v_{1} l+C_{1} \cos \beta_{1} l+D_{1} \sin \beta_{1} l\right)=0
\end{gathered}
$$

and

$$
\begin{gathered}
-\left(\eta N+E I_{1}\right) v_{1}^{3}\left(A_{1} \sinh v_{1} l+B_{1} \cosh v_{1} l\right)-\beta_{1}^{3}\left(-C_{1} \sin \beta_{1} l+D_{1} \cos \beta_{1} l\right) \\
-\mu \eta \omega^{2}\left(A_{1} \sinh v_{1} l+B_{1} \cosh v_{1} l-C_{1} \sin \beta_{1} l+D_{1} \cos \beta_{1} l\right)=0 .
\end{gathered}
$$

Since the system (57)-(62) is a linear homogenous system with respect to $A_{1}, B_{1}, C_{1}, D_{1}, A_{0}, B_{0}$, its determinant $\Delta$ must vanish. The equation $\Delta=0$ is solved with respect to the eigenfrequency $\omega$. Let us denote the row vectors of the determinant $\Delta$ by $\vec{a}_{j} ; j=1, \ldots, n$. It can be revealed from (57)-(62) that 


$$
\begin{gathered}
\vec{a}_{1}=\left(\cosh v_{1} a, \sinh v_{1} a, \cos \beta_{1} a,\right. \\
\left.\sin \beta_{1} a,-\cosh v_{0} a+\cos \beta_{0} a,-\sinh v_{0} a+\frac{v_{0}}{\beta_{0}} \sin \beta_{0} a\right) ; \\
\vec{a}_{2}=\left(v_{1} \sinh v_{1} a+C\left(\eta_{1}+v_{1}^{2} n_{1} \cosh v_{1} a\right),\right. \\
v_{1} \cosh v_{1} a+C\left(\eta_{1}+v_{1}^{2} n_{1} \sinh v_{1} a\right), \\
-\beta_{1} \sin \beta_{1} a+C\left(\eta_{1}-\beta_{1}^{2} n_{1} \cos \beta_{1} a\right), \\
\beta_{1} \cos \beta_{1} a+C\left(\eta_{1}-\beta_{1}^{2} n_{1} \sin \beta_{1} a\right), \\
\left.-v_{0} \sinh v_{0} a-\beta_{0} \sin \beta_{0} a,-v_{0}\left(\cosh v_{0} a-\cos \beta_{0} a\right)\right) ; \\
\vec{a}_{3}=\left(\left(v_{1}^{2} n_{1}+\eta_{1}\right) \cosh v_{1} a,\left(v_{1}^{2} n_{1}+\eta_{1}\right) \sinh v_{1} a,\right. \\
\left(-\beta_{1}^{2} n_{1}+\eta_{1}\right) \cos \beta_{1} a,\left(-\beta_{1}^{2} n_{1}+\eta_{1}\right) \sin \beta_{1} a, \\
-n_{0}\left(v_{0}^{2} \cosh v_{0} a+\beta_{0}^{2} \cos \beta_{0} a\right)-\eta_{0}\left(\cosh v_{0} a-\cos \beta_{0} a\right), \\
\left.\left.\vec{a}_{0} \sinh v_{0} a+\beta_{0} \sin \beta_{0} a\right)-\eta_{0}\left(\sinh v_{0} a-\frac{v_{0}}{\beta_{0}} \sin \beta_{0} a\right)\right) ; \\
\vec{a}_{6}=\left(v_{1}^{3} \sinh v_{1} l, v_{1}^{3} \cosh v_{1} l, \beta_{1}^{3} \sin \beta_{1} l,-\beta_{1}^{3} \cos \beta_{1} l, 0,0\right) \\
\vec{a}_{1}\left(v_{1}\left(n_{1} v_{1}^{2}+\eta_{1}\right) \sinh v_{1} a, v_{1}\left(n_{1} v_{1}^{2}+\eta_{1}\right) \sin \beta_{1} a, \beta_{1}\left(-n_{1} \beta_{1}^{2}+\eta_{1}\right) \cosh \beta_{1} a\right. \\
-n_{0}\left(v_{0}^{3} \sinh v_{0} a+\beta_{0}^{3} \sin \beta_{0} a\right)-\eta_{0}\left(v_{0} \sinh v_{0} a-\beta_{0} \sin \beta_{0} a\right) \\
\left.n_{0}\left(v_{0}^{2} \cosh v_{0} a+\beta_{0}^{2} \cos \beta_{0} a\right)-v_{0} \eta_{0}\left(\cosh v_{0} a-\cos \beta_{0} a\right)\right) ; \\
\left.v_{1}^{2} \sinh v_{1} l,-\beta_{1}^{2} \cos \beta_{1} l,-\beta_{1}^{2} \sin \beta_{1} l, 0,0\right) ;
\end{gathered}
$$

\section{NUMERICAL RESULTS}

The results of the calculations are presented in Figs 1-6 for cantilever nano-beams with a single step at $x=a$. The curves in Figs $1-6$ correspond to nano-beams of length $\mathrm{l}=1000 \mathrm{~nm}$, width $\mathrm{b}=50 \mathrm{~nm}$ and height $h_{0}=200 \mathrm{~nm}$. The Young modulus of the material is $\mathrm{E}=117 \mathrm{GPa}$ and the Poisson ratio $v=0.36$.

In Fig. 1 the dependence of the eigenfrequency on the crack length is demonstrated. Here $\Upsilon=h_{1} / h_{0}=$ 1.25. Different curves in Fig. 1 correspond to the cases where $a=0.3 l ; a=0.5 l$ and $a=0.7 l$, respectively. It can be seen from Fig. 1 that if the crack length increases, then the natural frequency will decrease. 


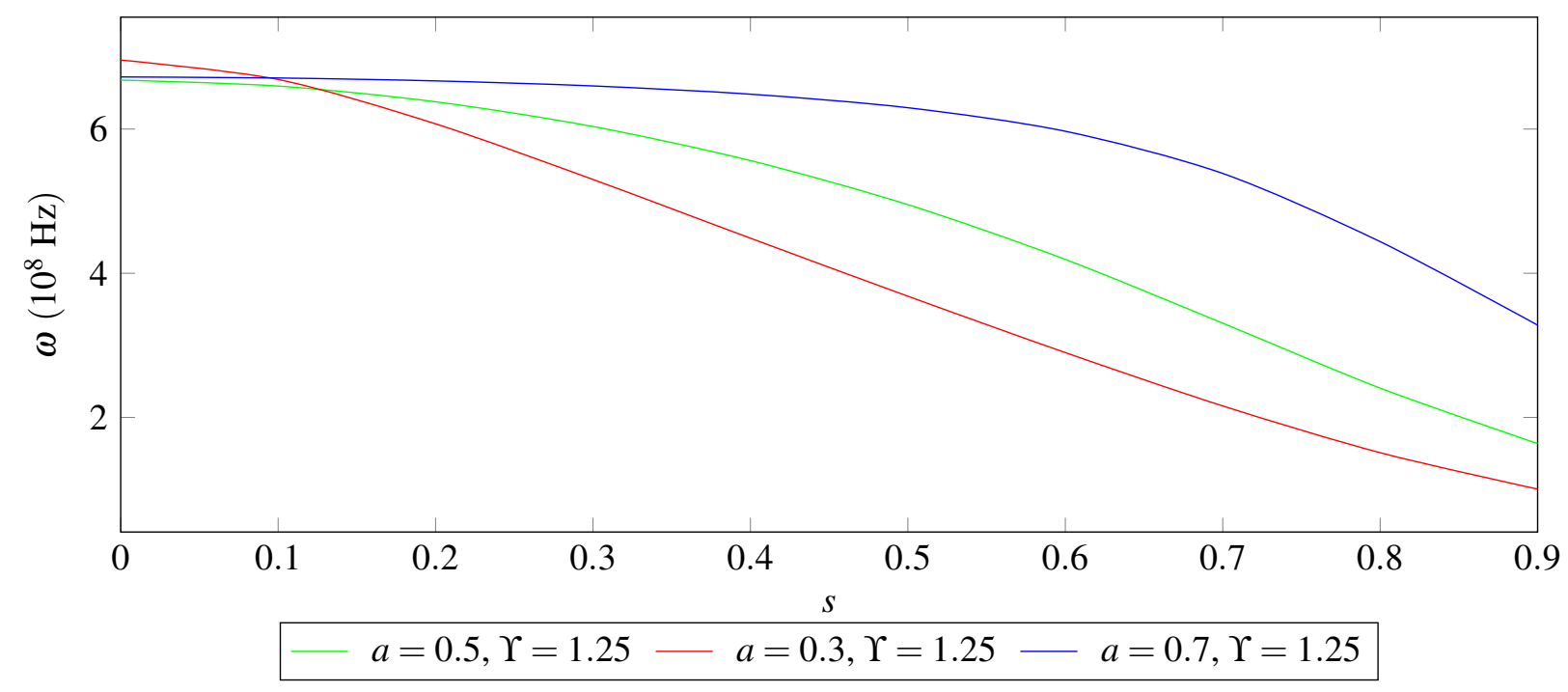

Fig. 1. Effect of $s$ by $a$.

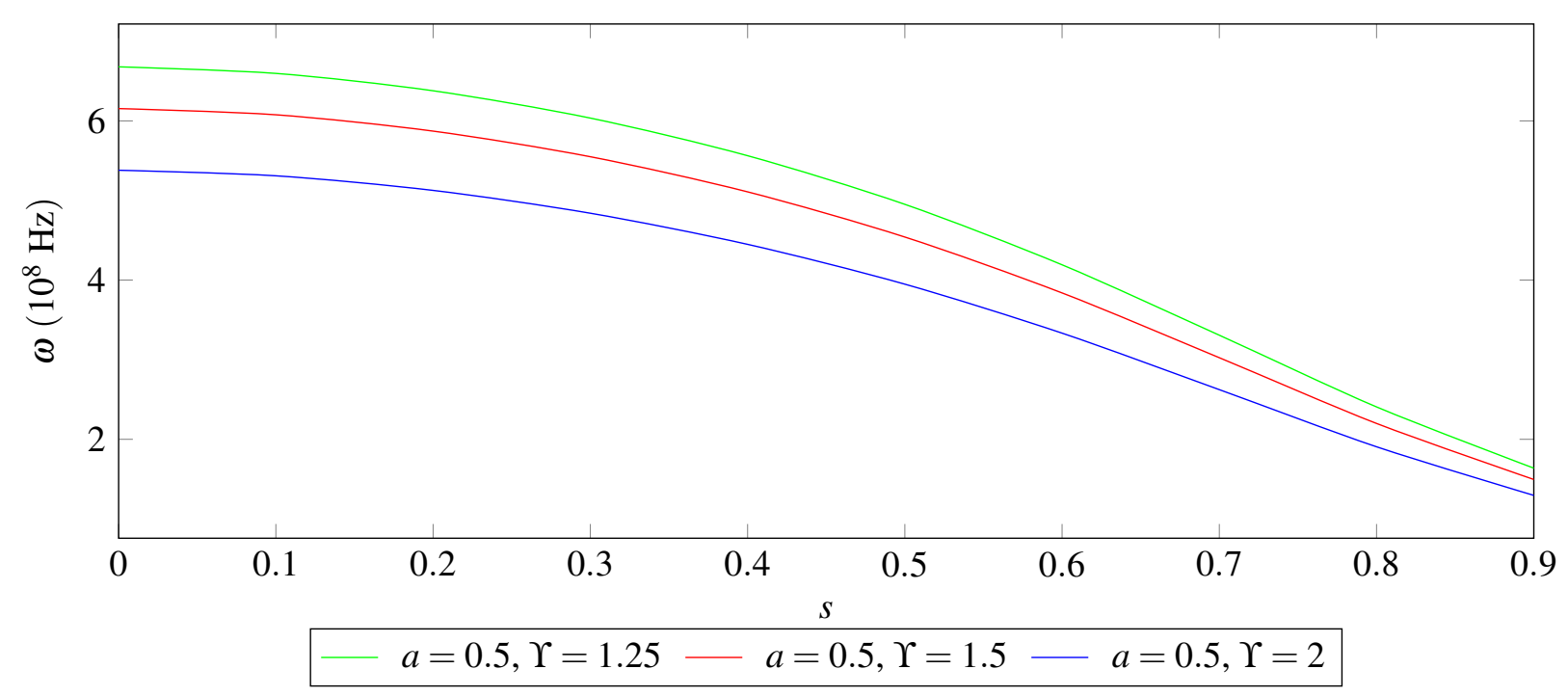

Fig. 2. Effect of $s$ by $\Upsilon$.

In Fig. 2 the natural frequency vs the ratio $c / h_{1}$ is shown for different values of $h_{1} / h_{0}$. Here $\Upsilon=1.25$; $\Upsilon=1.5 ; \Upsilon=2$ and $a=0.5 l$. It can be seen from Fig. 2 that the higher is the ratio $\Upsilon$, the lower is the natural frequency.

The relation between the natural frequency $\omega$ and the step location $a$ is demonstrated in Figs 3 and 4 .

Figure 3 depicts the nano-beam $\Upsilon=h_{1} / h_{0}=1.25$ and different curves are associated with cracks $c=0.3 h_{1} ; c=0.4 h_{1}$ and $c=0.6 h_{1}$. One can see from Fig. 3 that the lowest curve corresponds to the longest crack in the nano-beam and the highest curve is associated with the shortest crack. 


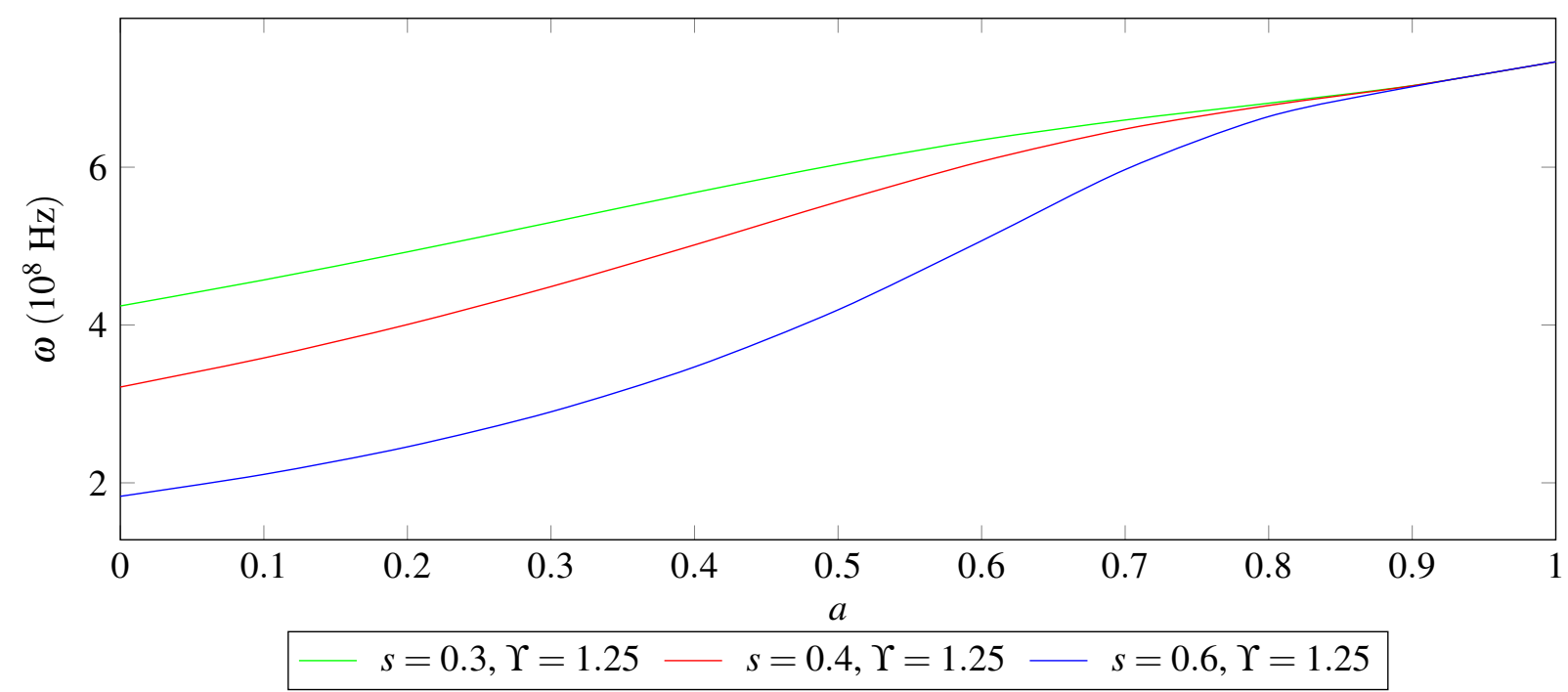

Fig. 3. Effect of $a$ by $s$.

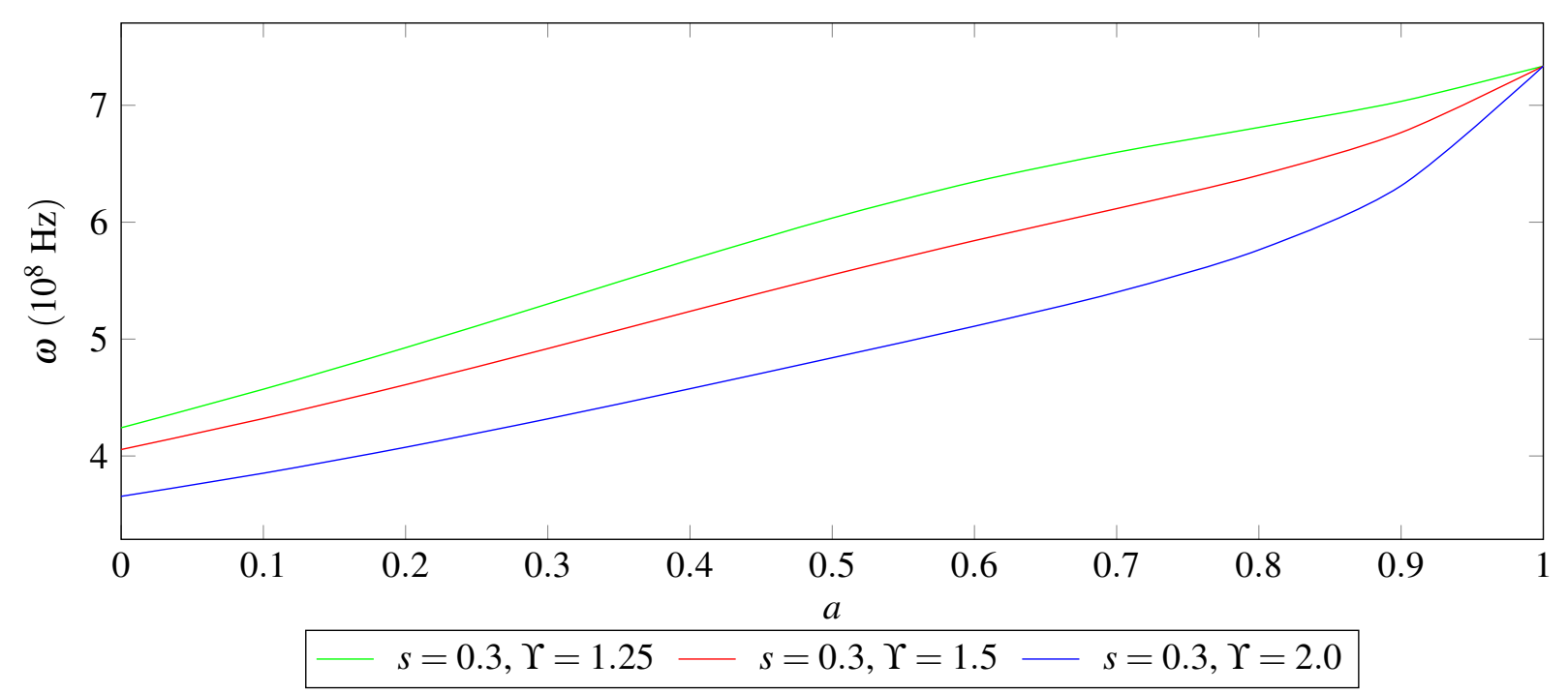

Fig. 4. Effect of $a$ by $\Upsilon$.

Figure 4 shows that in the case of the common crack length, the lowest natural frequencies correspond to the nano-beam with the highest ratio $\Upsilon=h_{1} / h_{0}$. The eigenfrequencies of nano-beams made of different materials are presented in Figs 5 and 6. In these figures the blue lines refer to titanium, red lines to nickel and green lines to copper. It can be seen from Figs 5 and 6 that the highest eigenfrequencies correspond to nano-beams made of titanium and the lowest ones to those made of copper. 


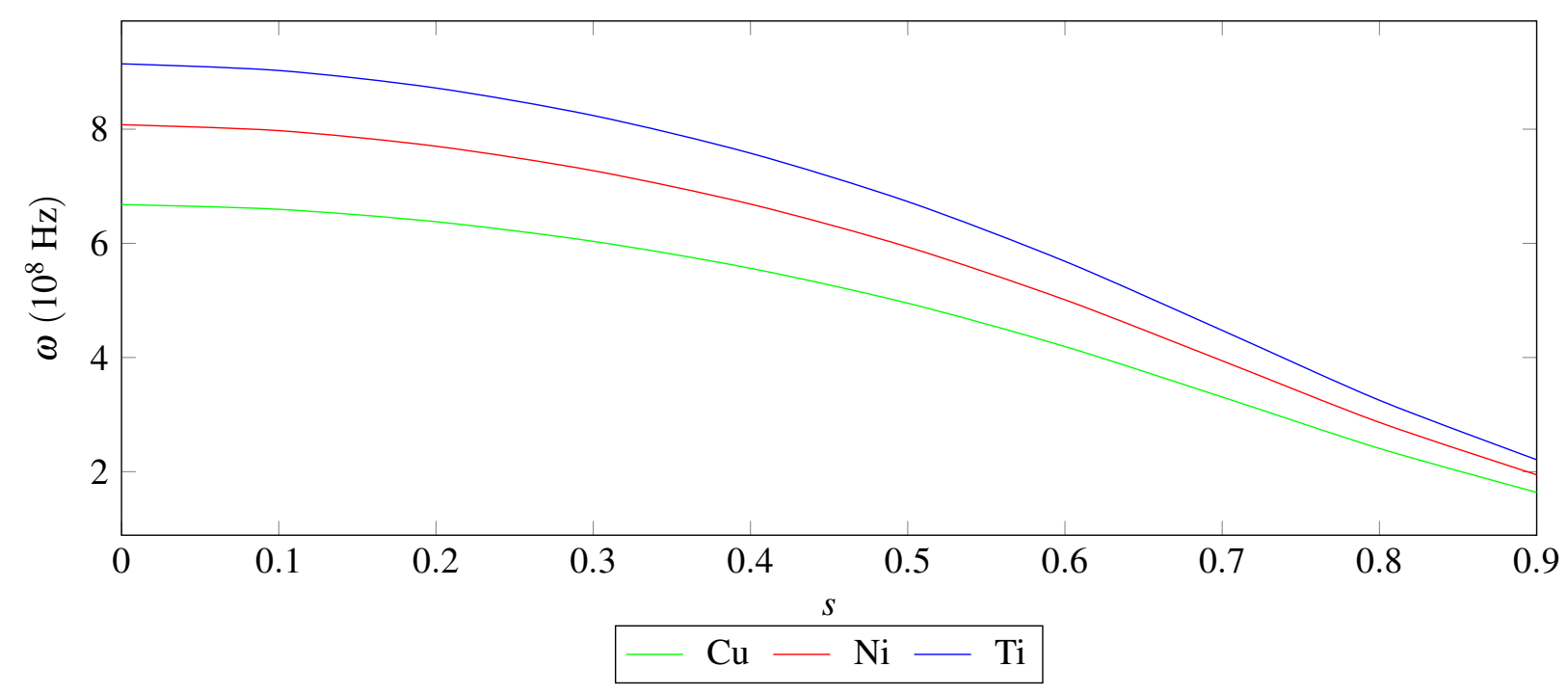

Fig. 5. Effect of $s$ by material.

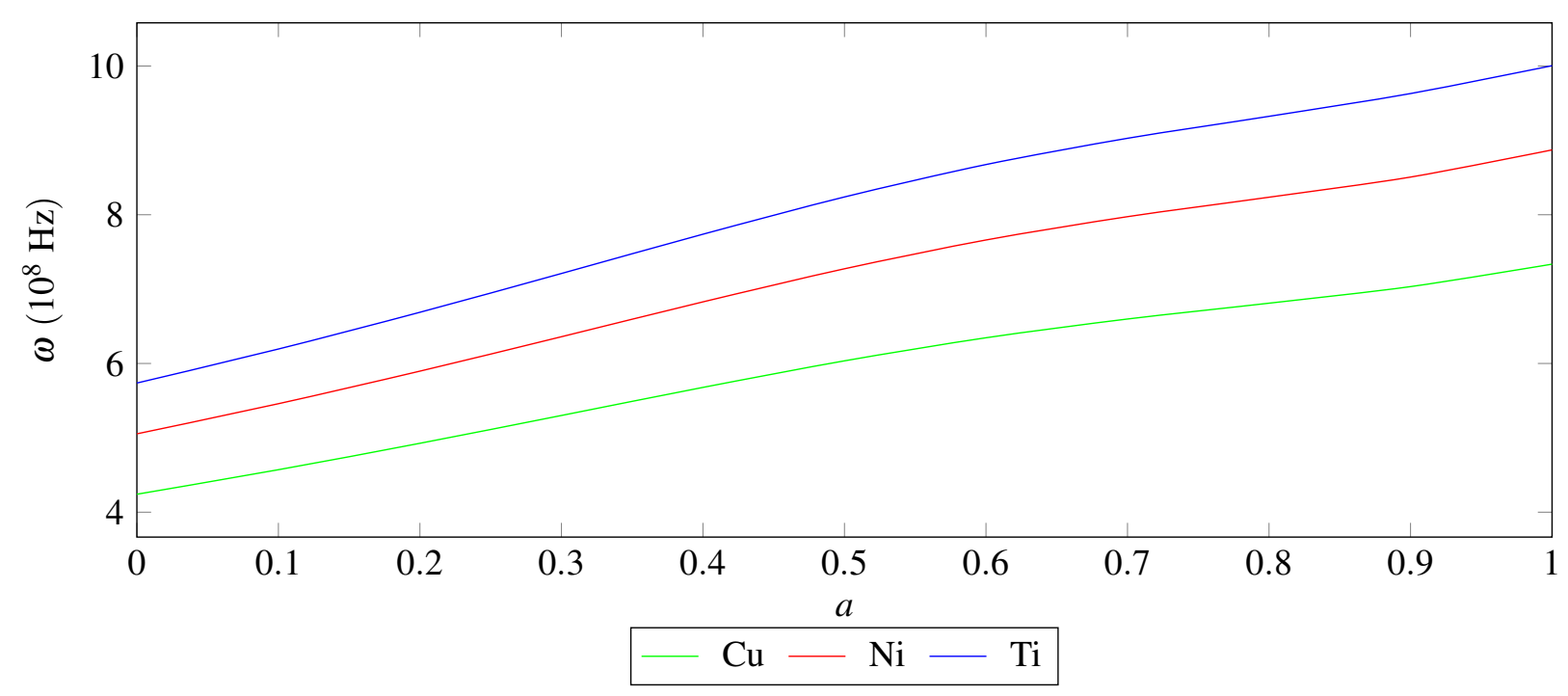

Fig. 6. Effect of $a$ by material.

\section{CONCLUSIONS}

A method for the vibration analysis of nano-beams has been developed. The nano-beams under consideration have stepped cross sections and are weakened with stable cracks.

The calculations performed show that the cracks affect essentially the natural frequencies of nanobeams. The calculations also reveal that in the case of stepped nano-beams, the lowest values of eigenfrequencies correspond to nano-beams with the deepest cracks. Certainly, this conclusion remains valid for nano-beams with constant thickness, as well. 


\section{ACKOWLEDGEMENTS}

The partial support from the Institutional Research Funding IUT 20-57 of the Estonian Ministry of Education and Research is gratefully acknowledged. The publication costs of this article were covered by the Estonian Academy of Sciences.

\section{REFERENCES}

1. Adali, S. Variational formulation for buckling of multi-walled carbon nanotubes modelled as nonlocal Timoshenko beams. $J$. Theor. Appl.Mech., 2012, 50(1), 321-333.

2. Anderson, T. L. Fracture Mechanics. Fundamentals and Applications. CRC Press, Boca Raton, FL, 2005.

3. Anifantis, N. and Dimarogonas, A. Stability of columns with a single crack subjected to follower and vertical loads. Int. J. Solids Struct., 1983, 19(4), 281-291.

4. Broek, D. The Practical Use of Fracture Mechanics. Kluwer Academic Publishers, Dordrecht, 1989.

5. Caddemi, S. and Caliò, I. The influence of the axial force on the vibration of the Euler-Bernoulli beam with an arbitrary number of cracks. Arch. Appl. Mech., 2012, 82(6), 827-839.

6. Challamel, N. On the comparison of Timoshenko and shear models in beam dynamics. J. Eng. Mech., 2006, 132(10), 11411145.

7. Challamel, N. Higher-order shear beam theories and enriched continuum. Mech. Res. Commun., 2011, 38(5), 388-392.

8. Challamel, N. and Elishakoff, I. Surface stress effects may induce softening: Euler-Bernoulli and Timoshenko buckling solutions. Physica E, 2012, 44(9), 1862-1867.

9. Chondros, T. G., Dimarogonas, A. D. and Yao, J. A continuous cracked beam vibration theory. J. Sound Vib., 1998, 215(1), $17-34$.

10. Dimarogonas, A. D. Vibration of cracked structures: A state of the art review. Eng. Fract. Mech., 1996, 55(5), 831-857.

11. Eringen, A. C. Nonlocal Continuum Field Theories. Springer, New York, NY, 2002.

12. Ghannadpour, S. A. M., Mohammadi, B. and Fazilati, J. Bending, buckling and vibration problems of nonlocal Euler beams using Ritz method. Compos. Struct., 2013, 96, 584-589.

13. Hellan, K. Introduction to Fracture Mechanics. McGraw-Hill, New York, NY, 1984.

14. Lellep, J. and Kraav, T. Buckling of beams and columns with defects. Int. J. Struct. Stab. Dyn., 2016, 16(8), 2550048.

15. Lellep, J. and Kägo, E. Vibrations of elastic stretched strips with cracks. Int. J. Mech., 2011, 5(1), 27-34.

16. Lellep, J. and Lenbaum, A. Natural vibrations of a nano-beam with cracks. Int. J. Theor. Appl. Mech., 2016, 1(1), $247-252$.

17. Lu, P., Lee, H. P. and Lu, C. Dynamic properties of flexural beams using a nonlocal elasticity model. J. Appl. Phys., 2006, 99, 073510.

18. Pradhan, S. C. and Phadikar, J. K. Nonlocal elasticity theory for vibration of nanoplates. J. Sound Vib., 2009, 325, $206-223$.

19. Reddy, J. N. Nonlocal theories of bending, buckling and vibration of beams. Int. J. Eng. Sci., 2007, 45(2-8), $288-307$.

20. Roostai, H. and Haghpanahi, M. Vibration of nanobeams of different boundary conditions with multiple cracks based on nonlocal elasticity theory. Appl. Math. Model., 2014, 38(3), 1159-1169.

21. Soedel, W. Vibrations of Shells and Plates. Marcel Dekker, New York, NY, 2004.

22. Tada, H., Paris, P. C. and Irwin, G. R. The Stress Analysis of Cracks Handbook. ASME Press, New York, NY, 2000.

23. Wang, C. M., Zhang, Y. Y., Ramesh, S. S. and Kitipornchai, S. Buckling analysis of micro- and nanorods/tubes based on nonlocal Timoshenko beam theory. J. Phys. D: Appl. Phys., 2006, 39, 3904-3909.

24. Zhou, L. and Huang, Y. Crack effect on the elastic buckling behavior of axially and eccentrically loaded columns. Struct. Eng. Mech., 2006, 22(2), 169-184.

\section{Pragudega nõrgestatud astmeliste nanotalade vabavõnkumised}

\section{Jaan Lellep ja Artur Lenbaum}

Uuritakse nanotalade vabavõnkumisi eeldusel, et nende paksus on astmeliselt muutuv ning et astmete nurkades asuvad stabiilsed praod või prao-tüüpi defektid. Nanotalade liikumise kirjeldamiseks kasutatakse Eringeni mittelokaalse elastsusteooria võrrandeid geomeetriliselt lineaarses seades. Prao mõju nanotala omavõnkumistele hinnatakse lokaalse järeleandlikkuse koefitsiendi abil. Viimane on seotud purunemise mehaanikas kasutatava pinge intensiivsuse koefitsiendiga prao tipu juures. Numbrilised tulemused on esitatud ühe astmega nanotalade jaoks. Näidatakse, et tulemused on heas kooskõlas kirjandusest leitavate teiste autorite töödega konstantse paksuse korral. 